\title{
Comparative Study on the Adsorption Capacity of Activated Carbon Prepared from Lapsi Seed Stone and Betel nut using Phosphoric Acid
}

\author{
Sahira Joshi \\ Department of Engineering science and Humanities \\ Institute of Engineering, Tribhuvan University, Lalitpur, Nepal \\ Corresponding author:sjoshi61@hotmail.com
}

Received: April 6, $2017 \quad$ Revised: July 17, $2017 \quad$ Accepted: July 25, 2017

\begin{abstract}
This paper presents the comparative study on the adsorption capacity of activated carbons prepared from Lapsi (Choerospondias axillaris) seed stone and Betel (Areca catechu) nut. Activated carbons (ACs) were prepared from Lapsi seed stone (LSS) and Betel Nut (BN) by chemical activation with $\mathrm{H}_{3} \mathrm{PO}_{4}$ (in the ratio of $1: 1$ by weight) at $400^{\circ} \mathrm{C}$. The pore structure of activated carbons was determined by iodine number and methylene blue number. Surface morphology of ACs was studied by scanning electron microscopy (SEM). Surface functional groups were analyzed by Fourier Transform Infra Red Spectroscopy (FTIR). As indicated by TGA analysis, the appropriate temperature required for carbonization was $400{ }^{\circ} \mathrm{C}$. Betel nut AC showed high iodine number and methylene number of $888 \mathrm{mg} / \mathrm{gm}$ and $369 \mathrm{mg} / \mathrm{gm}$ respectively. SEM micrographs of Betel nut AC show the presence of well developed pores on its surface. FTIR result indicated that both ACs contain $-\mathrm{OH},>\mathrm{C}=\mathrm{O}$ groups as oxygen containing surface functional groups. Based on the result, the $\mathrm{AC}$ prepared from betel nut by activation with $\mathrm{H}_{3} \mathrm{PO}_{4}$ is comparable with commercial activated carbon and could be used as potential adsorbent for removal of pollutants from water and waste water.
\end{abstract}

Keywords: Lapsi seed stone, Betel nut, phosphoric acid activated carbon

\section{Introduction}

Activated carbon (AC) is a predominantly amorphous solid carbon material with highly surface area, microporous structure and high degree of surface functional groups. These unique characteristic properties make $\mathrm{AC}$ a versatile adsorbent for the removal of organic and inorganic pollutants from water and waste water. Adsorption capacity of AC is highly influenced by the preparation conditions such as activation condition, activating agent and nature of precursor [11].

Activated carbon can be generally prepared by two different methods (i) Physical activation and (ii) chemical activation. Physical activation involves carbonization of precursor followed by the activation of the carbonized product at high temperatures in presence of oxidizing gases such as $\mathrm{CO}_{2}$ and steam. In chemical activation, the precursor is carbonized at low temperatures in presence of chemical agent under inert atmosphere. Chemical activation has been reported as more 
advantageous over physical activation because it requires relatively lower temperature and shorter time for activating the material. In addition, very high surface area $\mathrm{AC}$ can be obtained. The most commonly used chemical activating agents are $\mathrm{H}_{3} \mathrm{PO}_{4}, \mathrm{ZnCl}_{2}$, and $\mathrm{KOH}$. Among these, $\mathrm{H}_{3} \mathrm{PO}_{4}$ is widely used for activation of lignocellulosic materials.

Activated carbons have been commonly prepared from variety of carbonaceous materials such as wood, coconut shell, coal. The demand for AC has been increased due to the increased utilization of the carbon in pollution control. As a result, cost of $\mathrm{AC}$ is also growing depending on the application. Hence, there is a need for the sorting out new precursors for the preparation of AC which should be cost effective in comparison to the commercially available AC. Recently, use of agricultural wastes as an alternate precursor for the preparation of $\mathrm{AC}$ has notably increased as it is widely available at low cost. Presently, the use of coconut shell [9], Bamboo [1], olive oil stone [5], peach stone [10] etc has been reported in literature as a precursor for $\mathrm{AC}$ production.

In present study, Lapsi (Choerospondias axillaris) seed stone and Betel (Areca catechu) nut were selected as AC precursors because of their wide availability in Nepal. Moreover, little work has been reported on the production of AC using Lapsi seed stone and Betel nut. So Lapsi seed stone and betel nut is of special interest towards preparation of AC. This study focuses on to make comparison in the adsorption capacity of the AC from Lapsi seed stone and Betel nut by $\mathrm{H}_{3} \mathrm{PO}_{4}$ activation.

\section{Experimental}

\subsection{Materials}

The Lapsi and Betel nut were collected from vegetable market at Kalimati, Kathmandu. Lapsi seed stone and Betel nut were first washed with tap water then with distilled water, dried in an oven at $110{ }^{\circ} \mathrm{C}$ for 12 hours. The dried materials were crushed with mortar and electric grinder. The crushed particles are sieved into the fraction of size $312 \mu \mathrm{m}$. Chemicals used for this analysis were all analytical grade purity and the solutions were prepared in distilled water. The nitrogen gas is of ultra high pure (UHP) grade.

\subsection{Preparation of Activated Carbon}

The Lapsi seed stone (LSS) and Betel nut (BN) powder were separately mixed with $\mathrm{H}_{3} \mathrm{PO}_{4}$ in the ratio of $1: 1$ by weight. Then the mixture was heated in hot plate till dry mass was obtained. Then, the samples were oven dried at $110^{\circ} \mathrm{C}$ for 24 hours. The dry mass was separately transferred to a quartz tube and placed in horizontal tubular furnace (Accumax, India) and carbonized at temperature of $400^{\circ} \mathrm{C}$ for 3 hours in continuous flow of nitrogen gas at flow rate of $100 \mathrm{~mL} /$ minute. The product was cooled to room temperature. Then treated with $0.1 \mathrm{M} \mathrm{NaOH}$ and washed with distilled water till free from acid. The resulting carbons were then dried in an electric oven at $110^{\circ} \mathrm{C}$ for 3 hours. The well dried ACs were sieved into the particle size of $106 \mu \mathrm{m}$ and used for further investigations.

\subsection{Characterization of Precursor}

In order to predict thermal behavior of the precursor, TGA analysis of Lapsi seed stone and Betel nut was carried out. Thermo gravimetric analysis (TGA) was carried out by a thermo gravimetric analyzer (EXSTAR 6300 SEIKO TG/DTA, Korea). 


\subsection{Characterization of Activated Carbon}

Characterizations of ACs were performed by determining iodine number, methylene blue number, scanning electron microscopy (SEM) and fourier Transform-Infrared (FTIR) Spectroscopy.

\subsubsection{Determination of Iodine Number}

Iodine number $\left(\mathrm{I}_{\mathrm{N}}\right)$ indicates the extent of micro pore distribution in the carbon. It is defined as the milligram of iodine adsorbed by $1.0 \mathrm{gm}$ of carbon [4]. The iodine number correlates with surface area. The iodine number was determined according to the standard method (ASTM method, 2006). In this method, $100 \mathrm{mg}$ of activated carbon was added to $5 \mathrm{~mL}$ of $5 \% \mathrm{HCl}$ and then boiled and cooled. To the cooled solution $10 \mathrm{~mL}$ of $0.1 \mathrm{~N}$ iodine solution was added, shaken for 30 second and filtered. The filtrate was titrated with $0.1 \mathrm{~N}$ sodium thiosulphate solution using starch as an indicator. Iodine number is determined by the following equation.

$$
\text { Iodinenumber }=\frac{\text { Weight of activatedcarbon }(\mathrm{mg})}{\text { Weight of activatedcarbon }(\mathrm{gm})}
$$

\subsubsection{Determination of Methylene blue number}

Methylene blue number $\left(\mathrm{MB}_{\mathrm{N}}\right)$ indicates the extent of meso pore distribution in the carbon. It is defined as the maximum amount of methylene blue (MB) dye adsorbed on 1.0 gm of carbon [4]. $\mathrm{MB}_{\mathrm{N}}$ of the activated carbon was determined according to Method [8]. For the determination of methylene blue number, $0.1 \mathrm{gm}$ of $\mathrm{AC}$ was added to $100 \mathrm{ml}$ of $\mathrm{MB}$ of concentration $100 \mathrm{ppm}$ and then equilibrated for 3 hours at $200 \mathrm{rpm}$. Then the solution was filtered through filter paper Whatman 41, and the remaining concentration of methylene blue was determined by measuring the absorbance at $664 \mathrm{~nm}$ using a UV/Vis spectrophotometer (CECIL-CE-100).

Methylene blue number of the $\mathrm{AC}$ is calculated by equation (2).

$$
\mathrm{MB}_{\mathrm{N}}(\mathrm{mg} / \mathrm{gm})=\frac{\left(\mathrm{C}_{\mathrm{O}}-\mathrm{C}_{\mathrm{e}}\right) \times \mathrm{V}}{\mathrm{M}}
$$

Where $\mathrm{C}_{\mathrm{o}}(\mathrm{mg} / \mathrm{L})$ and $\mathrm{C}_{\mathrm{e}}(\mathrm{mg} / \mathrm{L})$ is the initial and equilibrium concentration of the methylene blue solution, $\mathrm{V}(\mathrm{L})$ is the volume of the solution and $\mathrm{M}(\mathrm{gm})$ is the mass of the AC.

\subsubsection{Fourier Transform-Infrared (FTIR) Spectroscopy}

FTIR spectra were recorded on the Thermo Electron Corporation, Nicolet 4700 at room temperature. The $\%$ of transmission of samples was recorded over $500-4000 \mathrm{~cm}^{-1}$.

\subsubsection{Scanning electron microscopy (SEM)}

Surface morphology of the AC samples was determined using scanning electron microscope U-8000, Hitachi Co. Ltd. Japan.

\section{Results and discussion}

3.1 Thermogravimetric Analysis (TGA): TGA graph of Lapsi seed stone and Betel nut is shown in Fig. 1 


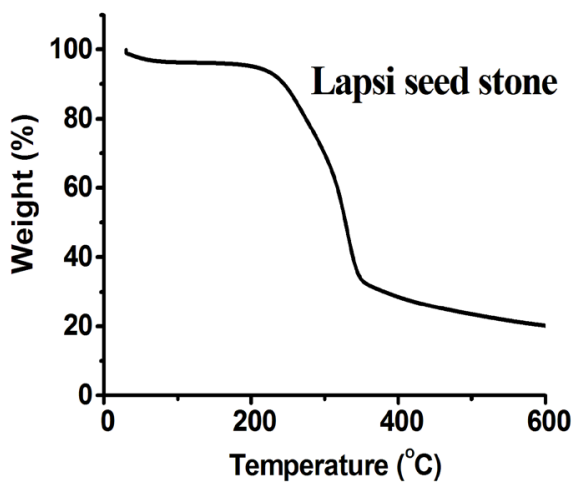

(a)

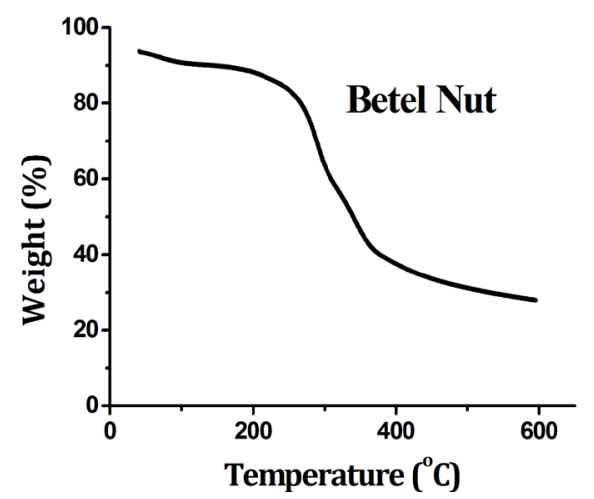

(b)

TGA graph of Lapsi seed stone

Fig. 1: TGA graphs of Lapsi seed stone and Betel nut

TGA is used primarily to determine the composition of materials and to predict their thermal stability up to elevated temperatures. As it can be seen in TG curve, thermal decomposition of both precursors Lapsi seed stone and Betel nut proceed through three major steps. The weight of the precursors decreases only slightly until $220^{\circ} \mathrm{C}$. It corresponds to the elimination of absorbed water and then a rapid weight loss is observed upto $380^{\circ} \mathrm{C}$. It might be caused by decomposition of cellulose, hemicellulose. A little loss of mass from 380 to $600^{\circ} \mathrm{C}$ corresponds to the decomposition of lignin. It indicates that, thermal decomposition of precursors was completed by $400{ }^{\circ} \mathrm{C}$. Hence $400^{\circ} \mathrm{C}$ was selected as the appropriate carbonization temperature for the preparation of activated carbons.

\subsection{Iodine Number and Methylene Blue Number}

Iodine and methylene blue adsorption is considered a simple and quick test for evaluating the porous structure of micro and mesoporous carbons. Iodine has a small molecular size and can readily penetrate deep micropores of the AC. So iodine number gives approximate measure of the micropore content of the carbon. Likewise, methylene blue number indicates the mesopore distribution in the carbon and indicates the ability of AC to adsorb medium size molecules. $\mathrm{I}_{\mathrm{N}}$, and $\mathrm{MB}_{\mathrm{N}}$ of $\mathrm{H}_{3} \mathrm{PO}_{4}$ activated LSS carbon and BN carbon is shown in Fig. 1. 


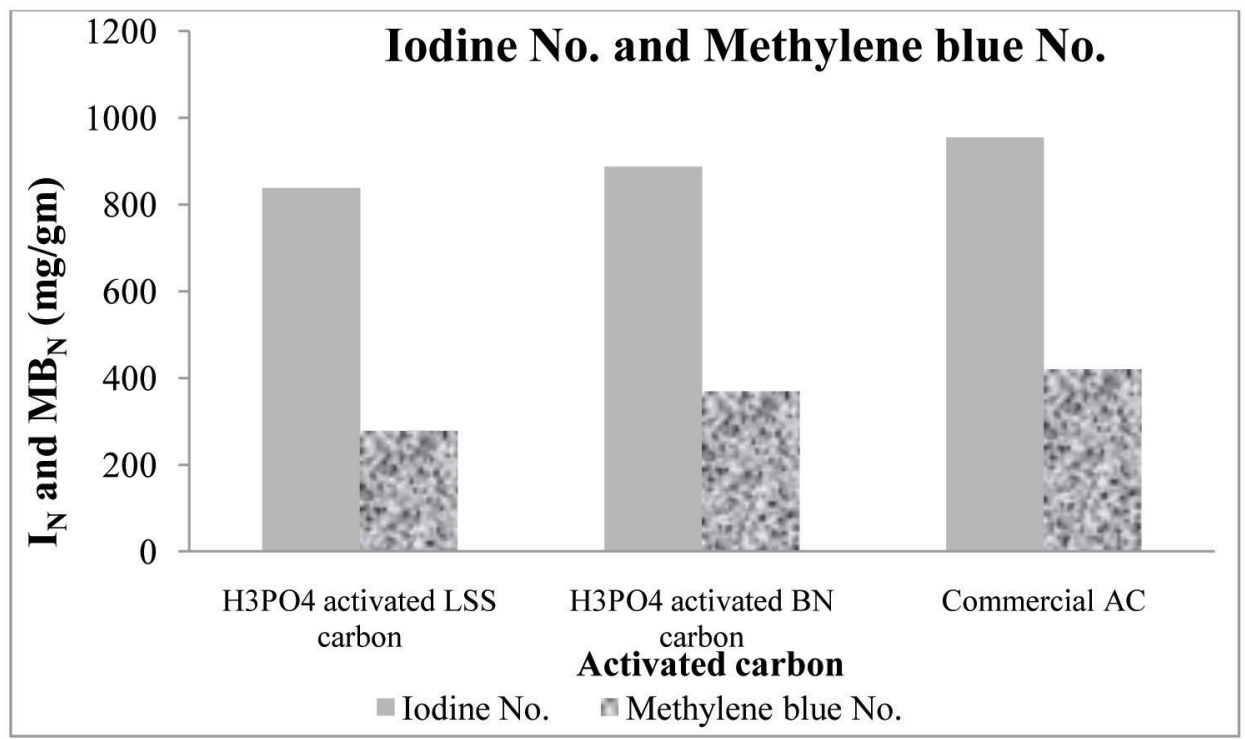

Fig. 2: $\mathrm{I}_{\mathrm{N}}$ and $\mathrm{MB}_{\mathrm{N}}$ of $\mathrm{H}_{3} \mathrm{PO}_{4}$ activated LSS and $\mathrm{BN}$ carbon

The iodine number and methylene blue number of the $\mathrm{H}_{3} \mathrm{PO}_{4}$ activated $\mathrm{BN}$ carbon was slightly higher than that of the LSS carbon. The values are comparable to commercial AC. It indicated that, $\mathrm{H}_{3} \mathrm{PO}_{4}$ activated LSS carbon is highly microporous and mesoporous in nature. Thus, botanical texture of the precursor affects the pore structure of $\mathrm{H}_{3} \mathrm{PO}_{4}$ activated LSS carbon significantly which directly links to its adsorption property.

\subsection{Scanning electron microscopy (SEM) image}

Scanning Electron Microscopy (SEM) was used to observe the surface morphology of AC. SEM images of the $\mathrm{H}_{3} \mathrm{PO}_{4}$ activated LSS carbon and BN carbon is shown in Fig. 2.

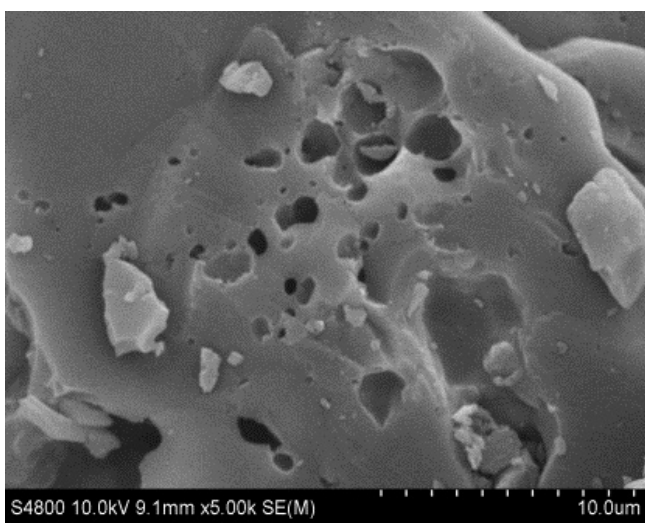

Fig. 2(a) $\mathrm{H}_{3} \mathrm{PO}_{4}$ activated LSS carbon

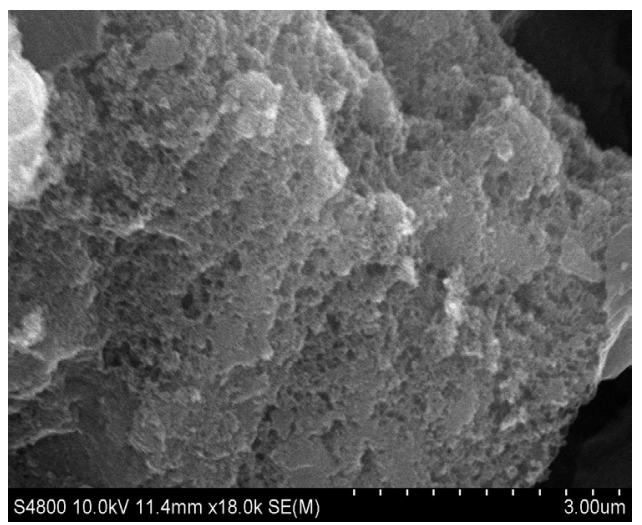

Fig. 2(b) $\mathrm{H}_{3} \mathrm{PO}_{4}$ activated $\mathrm{BN}$ carbon

Fig. 3: SEM Images of the $\mathrm{H}_{3} \mathrm{PO}_{4}$ activated LSS carbon and $\mathrm{H}_{3} \mathrm{PO}_{4}$ activated $\mathrm{BN}$ carbon

SEM images show that, both ACs contain numerous pores and crevices over their surface. $\mathrm{H}_{3} \mathrm{PO}_{4}$ activated LSS carbon contained open heterogeneous macropores on the external surface. The external surface of $\mathrm{H}_{3} \mathrm{PO}_{4}$ activated $\mathrm{BN}$ carbon also shows full of pores of different shape and 
sizes. It seems that the cavities on the surfaces of carbons resulted from the evaporation of the activating agent (which in this case is phosphoric acid) during carbonization, leaving the space previously occupied by the activating agent. $\mathrm{H}_{3} \mathrm{PO}_{4}$ functions in two ways during activation. As an acidic catalyst, it promotes bond cleavage reactions and forms crosslink in cellulose chains. Then, it combines with cellulose chains to form phosphate linkages, such as phosphate and polyphosphate esters, that can serve to connect and crosslink biopolymer fragments. $\mathrm{H}_{3} \mathrm{PO}_{4}$ activated $\mathrm{BN}$ carbon showed more pores and cavities on their external surface. Hence, it suggested that, surface morphology of the $\mathrm{H}_{3} \mathrm{PO}_{4}$ activated LSS carbon and $\mathrm{BN}$ carbon is influenced by botanical texture of the precursor material.

\subsection{Fourier Transform Infrared (FTIR) Spectra}

The adsorption capacity of AC depends upon porosity as well as the chemical reactivity of functional groups at the surface. Various functional groups present on the AC surface are responsible for preferential adsorption for different molecule. FTIR spectra of $\mathrm{H}_{3} \mathrm{PO}_{4}$ activated LSS carbon and BN carbon is shown in Fig. 3.

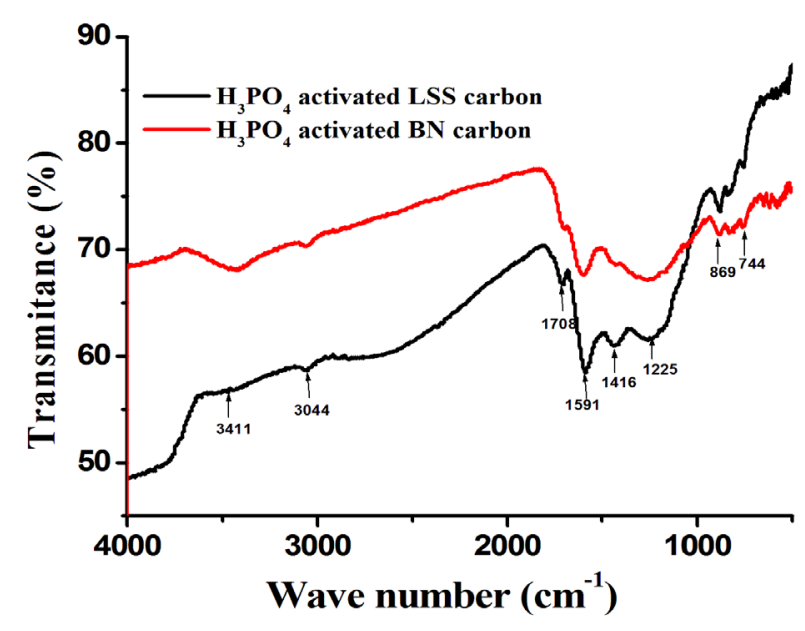

Fig. 4: FTIR of $\mathrm{H}_{3} \mathrm{PO}_{4}$ activated LSS carbon and $\mathrm{H}_{3} \mathrm{PO}_{4}$ activated $\mathrm{BN}$ carbon

FTIR spectra of $\mathrm{H}_{3} \mathrm{PO}_{4}$ activated LSS carbon and BN carbons were quite similar. Only slight differences on the intensity of the bands were detected. A broad band at around $3400 \mathrm{~cm}^{-1}$ indicated the presence of the $-\mathrm{OH}$ group of phenol. The band located at around $1700 \mathrm{~cm}^{-1}$ indicates $\mathrm{C}-\mathrm{O}$ stretching of carboxyl or carbonyl groups. The band around $1591 \mathrm{~cm}^{-1}$ and $1416 \mathrm{~cm}^{-1}$ is indicated to $\mathrm{C}=\mathrm{C}$ stretching vibration in aromatic skeleton generally found in carbonaceous material, such as activated carbon [3]. Both ACs contain oxygenated functional groups such as hydroxyl, carbonyl. It shows that, the nature of precursor has no significant effect on the surface functional groups of $\mathrm{H}_{3} \mathrm{PO}_{4}$ activated LSS and $\mathrm{BN}$ carbon.

\section{Conclusion}

Adsorption properties of the ACs prepared from Lapsi seed stone and Betel nut by chemical activation with $\mathrm{H}_{3} \mathrm{PO}_{4}$ was compared. Iodine and methylene blue adsorption indicates that $\mathrm{H}_{3} \mathrm{PO}_{4}$ activated Betel nut carbon is highly micro and mesoporous with high adsorption capacity. As 
shown by SEM micrograph, $\mathrm{H}_{3} \mathrm{PO}_{4}$ activated $\mathrm{BN}$ carbons shows well developed pore structure on its external surface. FTIR results indicate that both ACs contain oxygenated functional groups such as hydroxyl, carbonyl. The result of adsorption properties indicated that, $\mathrm{H}_{3} \mathrm{PO}_{4}$ activated Betel nut carbon can thus be applied for the removal inorganic and organic compounds from water and effluents by adsorption process.

Acknowledgement: The author would also like to express deepest gratitude to Prof. Dr. Raja Ram Pradhananga for research guidance and support.

\section{References}

[1] Ademiluyi FT and Nze JC (2016), Multiple Adsorption of Heavy Metal Ions in Aqueous Solution Using Activated Carbon from Nigerian Bamboo, International Journal of Research in Engineering and Technology, 5: 164-169.

[2] Annual Book of ASTM Standards (2006), Standard Test Method for Determination of Iodine Number of Activated Carbon, ASTM D4607-94, Philadelphia PA, United State of America.

[3] Bostancioglu M and Oruc S (2012), Preparation and Characterization of Activated Carbons from Furfural Production Wastes, Journal of Civil Engineering and Urbanism, 2 : 1-5.

[4] Cleiton NA and Guerreiro MC (2011), Estimation of surface area and pore volume of activated carbons by methylene blue and iodine numbers, Quimica Nova, 34 : 472-476.

[5] Fiol N, Villaescusa I, Martínez M, Miralles N, Poch J and Serarols J (2006), Sorption of $\mathrm{Pb}(\mathrm{II}), \mathrm{Ni}(\mathrm{II}), \mathrm{Cu}(\mathrm{II})$ and $\mathrm{Cd}(\mathrm{II})$ from Aqueous Solution by Olive Stone Waste, Separation and Purification Technology, $50: 132-140$.

[6] Gottipati RK (2012), Preparation and Characterization of Microporous Activated Carbon from Biomass and its Application in the Removal of Chromium(VI) from Aqueous Phase, PhD Thesis, Department of Chemical Engineering, National Institute of Technology, Rourkela, Odisha 769008, India.

[7] Lu JJ and Chen WH (2014), Product yields and characteristics of corncob waste under various torrefaction atmospheres, Energies, $7: 13-27$.

[8] Raposo F, De La Rubia MA and Borja R (2009), Methylene blue number as useful indicator to evaluate the adsorptive capacity of granular activated carbon in batch mode: Influence of adsorbate/adsorbent mass ratio and particle size, Journal of Hazardous Materials, 165 : 291299.

[9] Sathish RS, Raju NSR, Raju GS, Rao GN, Kumar KA and Janardhana C (2007), Equilibrium and kinetic studies for fluoride adsorption from water on Zirconium impregnated coconut shell carbon, Separation Science and Technology, $42: 769-788$.

[10] Tahvildari K, Bigdeli TS, Esfahani N and Farshchi M (2009), Optimization of Activated Carbon Preparation from Peach Stone, Journal of Applied Chemical Research, 11 : 47-55.

[11] Tan IAW, Ahmad AL and Hameed BH (2008), Adsorption of basic dye on high-surface-area activated carbon prepared from coconut husk: Equilibrium, kinetic and thermodynamic studies, Journal of Hazardous Materials, 154 : 337-346. 